19 Revue d'histoire du XIXe siècle

Société d'histoire de la révolution de 1848 et des

révolutions du XIXe siècle

22 | 2001

Autour de Décembre 1851

\title{
Le coup d'État du 2 décembre 1851. Bibliographie
}

Sylvie Aprile and Raymond Huard

\section{OpenEdition}

\section{Journals}

Electronic version

URL: http://journals.openedition.org/rh19/252

DOI: $10.4000 /$ rh19.252

ISSN: $1777-5329$

Publisher

La Société de 1848

Printed version

Date of publication: 1 June 2001

ISSN: 1265-1354

Electronic reference

Sylvie Aprile and Raymond Huard, «Le coup d'État du 2 décembre 1851. Bibliographie », Revue d'histoire du XIXe siècle [Online], 22 | 2001, Online since 04 September 2008, connection on 30 April 2019. URL : http://journals.openedition.org/rh19/252 ; DOI : 10.4000/rh19.252

This text was automatically generated on 30 April 2019

Tous droits réservés 


\title{
Le coup d'État du 2 décembre 1851. Bibliographie
}

\author{
Sylvie Aprile and Raymond Huard
}

1 La bibliographie que nos présentons ci dessous s'efforce de cerner au plus près ce qui a été écrit sur le Coup d'État du 2 décembre 1851 depuis l'événement jusqu'à nos jours. Toutefois pour éviter qu'elle ne prenne des dimensions disproportionnées, elle ne prend pas en compte - ou seulement de façon exceptionnelle - trois séries d'ouvrages dont l'importance pour une étude du coup d'État n'est pas négligeable. Il s'agit d'abord des histoires générales de la Seconde République dont certaines consacrent au coup d'État des pages substantielles, ensuite des biographies de Louis-Napoléon Bonaparte ou de Napoléon III, enfin des thèses ou travaux départementaux sur l'histoire de la Seconde République qui couvrent toute la période entre 1848 et 1851 sans s'attacher particulièrement au coup d'État. Pour éviter cependant d'en faire totalement abstraction, ce qui serait injustifié, nous avons ajouté à la fin de la bibliographie, une annexe qui rappelle de façon succincte quels sont les ouvrages fondamentaux de chacune de ces trois catégories en mentionnant seulement leur auteur et le domaine géographique couvert. Une partie de ces ouvrages figurent déjà dans la bibliographie de 1848 établie par les soins de notre société en 1998 et publiée dans le n¹4, 1997/1 de notre revue. Le lecteur pourra donc s'y reporter.

\section{HISTOIRES ET TÉMOIGNAGES IMMÉDIATS}

\section{Bonapartistes ou conservateurs}

A. BARBIER, Histoire de Louis-Napoléon Bonaparte, Président de la République française, depuis sa naissance jusqu'à ce jour, contenant l'appréciation de ses actes, le récit authentique de tous les événements du 2 décembre et des détails inédits sur ses habitudes privées, Paris, Barbier, 1852, in $18,180 \mathrm{p}$.

Ernest DU BARRAIL, Histoire de la Jacquerie de 1851, Paris, aux bureaux du Public, 1852, 80 p. Paul BELOUINO, Histoire d'un coup d'État (décembre 1851) d'après les documents authentiques, les pièces officielles et le renseignements intimes, Paris, L. Brunet \& cie, 1852, 492 p.

Henri BOSSELET, La Crise, Paris, Garnier frères, 1852, 190 p.

Louis-Napoléon BONAPARTE, Discours et Messages depuis son retour en France jusqu'au 
2 décembre 1852, Paris, Librairie Plon, 1853.

Charles BRUN, Récit des événements de décembre. Oui ou non!!! Appel à la nation Vote universel du 21 décembre 1851, Paris, Ledoyen, 1851.

Barnabé CHAUVELOT, La Restauration de l'autorité, Paris, Allouard et Kaeppelin, 1851, 94 p. Joseph DELAORA, Le Coup d'État, c'est l'avenir!, Paris, Garnier frères, 1851, 48 p. [10 décembre 1851]

Jean-Claude-Barthélémy GALLIX et GUY, de l'Hérault, Histoire complète et authentique de Louis-Napoléon Bonaparte, depuis sa naissance jusqu'à ce jour, précédée d'un avant propos intitulé le 2 décembre devant l'histoire, Paris, H. Morel, 1852, in $8^{\circ}, 502 \mathrm{p}$.

Henri LASERRE, L'opinion et le coup d'État, Paris, Charpentier, 1851.

LAURENT (de l'Ardèche), Coup d'œil philosophique sur la Révolution de décembre, Paris, Garnier, 1852, 16 p.

Henri LEMULLIER, Histoire parlementaire de la présidence depuis l'élection du prince Louis Napoléon Bonaparte jusqu'au 2 décembre 1851, Paris, Garnier frères, 1852, in 18, 230 p.

Léo LESPÈS, Histoire politique, anecdotique et philosophique de la première présidence du prince Louis Napoléon Bonaparte depuis le 10 décembre 1848 jusqu'au 20 décembre 1851, Paris, C. Ploche, 1852,2 volumes in $8^{\circ}$.

Hippolyte MAQUAN, L'Insurrrection de décembre 1851 dans le Var, Draguignan, Bernard, 1853, 279 p.

Hippolyte de MAUDUIT (capitaine), Révolution militaire du 2 décembre 1851 ; précédée de la vérité quand même à tous les partis, et de curieux entretiens de l'auteur avec le prince LouisNapoléon, Paris, A. Delahays, 1852, in 18, 304 p.

P. MAYER, Histoire du Deux Décembre: avec des documents inédits et des pièces justificatives, Paris, Ledoyen, 1852, in $12^{\circ}, 327$ p. ; 2e édition, Histoire du Deux décembre, Paris, Ledoyen, 1852, 355 p.

Prosper NOUBEL, Le Deux décembre, Agen, imprimerie P. Noubel, 1852, in folio, 3 p.

Esprit PRIVAT, Le doigt de Dieu. La situation avant et après le 2 décembre, Paris, Lévy frères, 1852 , in $18,107 \mathrm{p}$.

B. RENAULT, Histoire de la présidence et de la dictature de Louis Napoléon, précédée d'un précis sur le 18 brumaire et la 2 décembre, de détails curieux sur le nom et l'origine des Bonaparte, d'un tableau généalogique de la dynastie impériale et de ses alliances, etc., et rédigée sur des documents particuliers et authentiques, Paris, R. Ruel aîné, 1852 , in $8^{\circ}$, II-266 p.

Édouard TURQUETY, Les représentants en déroute ou le 2 décembre, poème en cinq chants, Paris, Ledoyen, 1852, $108 \mathrm{p}$.

Auguste VITU, Histoire de Napoléon III et du rétablissement de l'Empire, Paris, Ledoyen et Giret, 1854 , in $8^{\circ}, 516 \mathrm{p}$.

\section{Républicains ou socialistes}

Pierre Auguste CALLET, La voix mystérieuse, les proscrits, le scrutin du 20 décembre, la constitution de 1852, les conseillers de M. Bonaparte, Londres, Jeffs, 1852, in 32.

Jean Baptiste Adolphe CHARRAS (colonel), Enquête sur le 2 décembre et les faits qui le suivent, Bruxelles, chez tous les libraires, 1852, in 12, $54 \mathrm{p}$.

Jean Baptiste Adolphe CHARRAS (colonel), Les aides de camp du 2 décembre, extrait de La Nation, Amsterdam, Imprimerie de N.-P. Vandenbosche, 1853, in 16, 74 p.

Marc DUFRAISSE, Le Deux-Décembre devant le code pénal, Madrid, Impr. del Mundo literario, 1853 , in 64, $154 \mathrm{p}$.

Pascal DUPRAT, Les tables de proscription de Louis Bonaparte et de ses complices, Liège, Redouté, 1852, 2 volumes in $8^{\circ}, 318$ et $387 \mathrm{p}$.

Xavier DURRIEU, Le Coup d'État de Louis Bonaparte, Histoire de la persécution de décembre: 
événements, prisons, casemates et pontons, Londres/Genève/New York, J. Thomas, 1852, $217 \mathrm{p}$.

Victor HUGO, Napoléon le petit, Londres, Jeffs, 1852, 464 p.

Victor HUGO, Discours de M. Victor Hugo prononcé à Jersey sur la tombe d'un proscrit français le 20 avril 1853, suivi du discours de M. Bancel prononcé le même jour à Bruxelles sur la tombe d'un autre proscrit français, Bruxelles, 1853, in 16, $16 \mathrm{p}$.

Hippolyte MAGEN, Histoire de la terreur bonapartiste ; préliminaires et présages du coup d'État ; complément des débauches prétoriennes à Paris et dans les départements. bastilles, casemates et pontons ; la nouvelle Caprée, Londres, W. Jeffs, 1852, in 16, VIII-280 p.

Karl MARX, Der achzehnte brumaire des Louis Bonaparte - Die Revolution, $\mathrm{n}^{\circ} 1,1852$.

Pierre-Joseph PROUDHON, La Révolution sociale démontrée par le coup d'État du 2 décembre, Paris, Garnier frères, 1852, $283 \mathrm{p}$.

Charles RIBEYROLLES, Les bagnes d'Afrique, histoire de la transportation de décembre, Londres, Jeffs, 1853, $263 \mathrm{p}$.

Victor SCHCLCHER, Histoire des crimes du deux décembre, Londres, J. Chapman, 1852, $469 \mathrm{p}$.

Victor SCHCLCHER, Histoire des crimes du deux décembre. Édition considérablement augmentée, Bruxelles, chez tous les libraires, 1852, 2 volumes.

Théophile THORÉ, La restauration de l'autorité ou l'opération césarienne, par un ex-représentant du peuple, Bruxelles, J. Tarride, 1852, in 12, 24 p.

\section{MÉMOIRES, CORRESPONDANCES PUBLIÉES ULTÉRIEUREMENT}

François-Charles BARRAIL, Mes souvenirs, 1820-1879, Paris, Librairie Plon, 1894-1896, 3 volumes in $8^{\circ}$ [le coup d'État vu d'Algérie].

Jean Baptiste BOICHOT, Souvenirs d'un prisonnier d'État sous le Second Empire, Leipzig, Muquardt, 1867, in 12, $284 \mathrm{p}$.

Louis BONAPARTE (Prince), Loi d'enseignement, le deux décembre 1851, Paris, 1925, 393 p.

Albert DU CASSE (baron), Les dessous du Coup d'État. 1851, Paris, Albert Savine, 1891, in 18, $316 \mathrm{p}$.

Alfred de FALLOUX (comte), Mémoires d'un royaliste. Tome 2: La présidence du Prince Louis Bonaparte, Loi d'enseignement, le deux décembre 1851, Paris, Perrin, 1925, 393 p.

Fernand GIRAUDEAU, Le 2 décembre, Paris, Pérignon, 1873, 36 p.

François GUIZOT, Mémoires pour servir à l'histoire de mon temps, Paris, Michel Lévy, 1872, 8 volumes.

Pierre JOGNEAUX, Souvenirs historiques, Paris, Éditions Flammarion, 1891, 2 volumes in 12.

Martin NADAUD, Mémoires de Léonard, ancien garçon maçon, Bourganeuf, Duboueix, 1895, in $8^{\circ}$, VIII-512 $\mathrm{p}$.

KERRY (Lord), Le secret du coup d'État, correspondance inédite du Prince Louis Napoléon, de MM. Morny, de Flahaut et les autres, Évreux, E. Paul, 1928, 315 p.

Charlemagne Émile de MAUPAS, Mémoires sur le Second Empire, Paris, Dentu, 1884, 2 volumes in $8^{\circ}$.

Hippolyte MONIN, "Le coup d'État du 2 décembre à Montmartre", extrait de Le Vieux Montmartre, Paris, $1899,20 \mathrm{p}$.

Hippolyte MONIN, François Désiré Bancel, représentant de la Drôme, proscrit, professeur à Bruxelles, député de Paris, 1822-1871, Paris, É. Cornély et cie, 1911, in 8², 148 p.

MORNY (duc de), "La genèse d'un Coup d'État, Mémoire du duc de Morny publié par son petit fils", dans Revue des Deux Mondes, 1er décembre 1925, pp. 512-534.

Alexandre QUENTIN-BEAUCHART, Études et souvenirs sur la Deuxième République et sur le Second Empire (1848-1870), Paris, 1901-1902, 2 volumes, 484 et $622 \mathrm{p}$. 
Arthur RANC, Souvenirs, correspondance, 1831-1908, Paris, Cornély, 1913, in 16, 528 p.

George SAND, "Le coup d'État", dans Revue de Paris, 15 juin 1904, pp. 673-690.

Louis VÉRON, Nouveaux mémoires d'un bourgeois de Paris, depuis le 10 décembre 1848 jusqu'aux élections générales de 1863, le Second Empire, Paris, Librairie internationale, 1866, in 8 495 p. Horace de VIEL-CASTEL (comte), Mémoires du comte Horace de Viel-Castel sur le règne de Napoléon III (1851-1864), Paris, chez tous les libraires, 1883-1885, 6 volumes, tomes 1 et 2.

\section{OUVRAGES DES ANNÉES 1860 À 1880}

\section{Républicains}

anonyme, Le deux décembre, les massacres dans Paris, Paris, Bibliothèque démocratique, 1872 , in $32,191 \mathrm{p}$.

anonyme [Maurice ENGELHARD], L'Empire démasqué, Histoire abrégée des crimes de Louis Bonaparte et de ses complices. Première partie: l'usurpation du pouvoir, Londres/Bruxelles/ Genève, J. Harrison, 1863, 96 p.

Noël BLACHE, Histoire de l'insurrection du Var en décembre 1851, Paris, Lechevalier, 1869, $236 \mathrm{p}$.

Alphonse BRÉMOND, Histoire du coup d'État dans le département de la Haute-Garonne (1851-52), Toulouse, Hébrail Durand et cie, 1870, in 18, 180 p.

Pierre Théodore CHÉRON DE VILLIERS, Chapitre inédit de l'histoire du coup d'État, Limoges en 1851, Paris, Marteau, 1869, in 18, 35 p.

Joseph DÉCEMBRE et Edmond ALLONIER, Le Coup d'État du 2 décembre 1851 :historique des événements qui ont précédé le coup d'État, pièces et documents officiels, Paris, DécembreAllonier libraire-éditeur, 1868, $216 \mathrm{p}$.

Joseph DÉCEMBRE et Edmond ALLONIER, Histoire des conseils de guerre de 1852 ou précis des événements survenus dans les départements à la suite du coup d'État de décembre 1851, Paris, Décembre-Allonier libraire-éditeur, 1869, $467 \mathrm{p}$.

C. FRANC, Un proscrit de décembre, Cahors, Girma, 1871, in 12.

Benjamin GASTINEAU, Les Suites du coup d'État, dossier du Deux décembre, les transportés de décembre 1851, Paris, Librairie centrale, 1869, in 18, 277 p.

Léon GOUPY, Le Coup d'État dans la Mayenne. Juin 1870, Paris, Imprimerie de L. Goupy, 1871, $102 \mathrm{p}$.

Victor HUGO, Histoire d'un crime, Paris, Calmann-Lévy, 1877, 2 volumes.

Alexander William KINGLAKE, Histoire du 2 décembre 1851 et portrait historique de Napoléon III [...], Londres/Bruxelles/ New York, J. Chapman, 1867, in 16.

Georges LASSEZ, La vérité sur le deux décembre, Paris, Lechevalier, 1872, in 18, 34 p.

Pierre LEFRANC, Le Deux-Décembre, ses causes et ses suites, Paris, Lechevalier, 1870, in 18, $277 \mathrm{p}$.

Hippolyte MAGEN, Le Pilori. Liste par départements des proscripteurs de décembre 1851, Paris/ Bruxelles, chez tous les libraires, 1854-1871, in 16, 200 p.

F. MAZENC, Coup d'État du 2 décembre 1851 dans l'Aveyron, Albi, imprimerie. Nouguiès, 1872, $\mathrm{XVI}-112 \mathrm{p}$.

Alexandre PAPON, Chronique départementale, La République et le coup d'État dans le département de l'Eure, Paris, chez tous les libraires, 1869, in 18, $140 \mathrm{p}$.

Adolphe ROBERT, Statistique pour servir à l'histoire de l'insurrection du 2 décembre 1851, Paris et les départements, Paris, Librairie de la Renaissance, 1869, in 18, II-268 p.

J.-B. RAVOLD, Les transportés de la Meurthe en 1852, Paris, A. Degorce-Cadot, 1872, in $8^{\circ}$, VI-85 p.

A. ROGEARD, Le 2 décembre et la morale, suivie de l'Histoire du deux décembre, Francfort-sur- 
le-Main, 1866, XXIX-120 p.

Victor SCHœLCHER, Le crime de décembre en province, Paris, Bibliothèque démocratique, 1875 , in $32,191 \mathrm{p}$.

Alfred TALANDIER, Denis Dussoubs-Gaston, sa vie, sa mort (1818-1851), Paris, Imprimerie de J. Rigal, 1880, in 16, 35 p.

Eugène TÉNOT, La Province en décembre 1851, Étude historique sur le coup d'État, Paris, Lechevalier, 1865, VI-360 p.

Eugène TÉNOT, Paris en décembre 1851, Étude historique sur le coup d'État, Paris, Lechevalier, 1868, $236 \mathrm{p}$.

Arthur VERMOREL, Les hommes de 1851, Histoire de la Présidence et du rétablissement de l'Empire, Paris, 1869, Décembre-Allonnier libraire-éditeur, 1869, IV-424 p.

\section{Conservateurs}

Adolphe GRANIER DE CASSAGNAC, Histoire de la chute du roi Louis-Philippe, de la république de 1848 et du rétablissement de l'Empire (1847-1855), Paris, Librairie Plon, 1857, 2 volumes in $8^{\circ}$.

Adolphe GRANIER DE CASSAGNAC, Récit authentique des événements de décembre 1851 à Paris et dans les départements. Nouvelle édition précédée d'une introduction sur les coups d'État, Paris, Dentu, in 12, 1869, 144 p.

J. G. PRAT, Les Exploits du Deux-Décembre, récits d'histoire contemporaine. Première série. Les Deux revenants, la cité dévote, la Tour du Jaï, l'hôte du fermier, Paris, Lachaud, 1872, 189 p.

\section{OUVRAGES ET ARTICLES GÉNÉRAUX SUR LE COUP D'ÉTAT, POSTÉRIEURS À L'ANNÉE 1880}

Maurice AGULHON, "La résistance au coup d'État en province. Esquisse d'historiographie", dans Revue d'Histoire moderne et contemporaine, tome 21, janvier-mars 1974, pp. 18-26.

Octave AUBRY, Napoléon III à la conquête du pouvoir, Paris, Librairie Flammarion, 1934, in $12,128 \mathrm{p}$.

Émilie BERTRAND, La presse, les journalistes et le coup d'État de Louis-Napoléon Bonaparte, Mémoire de maîtrise sous la direction de Francis Démier, Université Paris 10, 1996.

Frédéric BLUCHE, [dir.], Le prince, le peuple et le droit. Autour des plébiscites de 1851 et 1852, Paris, Presses universitaires de France, 1999, 318 p.

Jean Baptiste BOCQUET, Napoléon III. Ses serments, son crime du 2 décembre, Paris, de Moquet, 1883 , in $18,16 \mathrm{p}$.

Jacques-Olivier BOUDON, "Baudin et la barricade du 3 décembre 1851: histoire et représentation de l'Empire à la République", dans Alain CORBIN et Jean-Marie MAYEUR [dir.], La Barricade, Paris, Publications de la Sorbonne, 1997, pp. 221-234.

Georges BOURGIN, "Les préfets de Napoléon III, historiens du coup d'État",dans Revue historique, tome 166, 1931, pp. 274-289.

Carlo BRONNE, "Le Rubicon", dans Revue des Deux Mondes, 1er décembre 1950.

Christophe CHARLE, "Le souvenir du Deux-décembre, les étudiants et le boulangisme", dans La France démocratique, mélanges offerts à Maurice Agulhon, Paris, Publications de la Sorbonne, 1998, pp. 277-286.

Adrien DANSETTE, Louis-Napoléon à la conquête du pouvoir, Paris, Librairie Hachette, 1961, $420 \mathrm{p}$.

Denise DEVOS, La Troisième République et la Mémoire du coup d'État de Louis Napoléon Bonaparte, Paris, Archives nationales, 1992.

Pierre DOMINIQUE [Pierre LUCCHINI], Louis-Napoléon et le Coup d'État du deux décembre, 
Paris, SFELT, 1951 , in $8^{\circ}$.

André ENCREVÉ, "Protestantisme et politique: Les protestants du Midi en décembre 1851", dans Droite et Gauche en Languedoc-Roussillon de 1789 à nos jours. Actes du colloque de Montpellier, 9-10 juin 1973, Montpellier, Centre d'histoire contemporaine du Languedoc méditerranéen et du Roussillon, 1975, 376 p., pp. 161-196.

Gisela GEYWITZ, Das Plebiszit von 1851 in Frankreich, Tubingen, Mohr, 1965, VIII-281 p.

Rémi GOSSEZ, "Bibliographie critique de la littérature du Coup d'État", dans 1848 et les révolutions du XIXe siècle, $\mathrm{n}^{\circ}$ 44, 1951, pp. 153-158.

Hamlaoui LAHRAKI, Le problème des grâces, au lendemain du coup d'État de décembre 1851 jusqu'à l'année 1853, Mémoire de maîtrise sous la direction de Philippe Vigier, Université Paris 10, 1984.

André LEBEY, Les trois coups d'État de Louis-Napoléon Bonaparte, Paris, Perrin, 1906, 519 p.

L. LEBRUN, L'indemnisation des victimes du coup d'État de 1851. Loi du 31 juillet 1881, Mémoire de maîtrise sous la direction de Maurice Agulhon, Université Paris 1, 1982.

Marianne LEULLIEZ, "La fortune romanesque des insurrections méridionales de décembre 1851", dans Roland ANDRÉANI et Marianne LEULLIEZ [dir.], De la Révolution au coup d'État (1848-1851). Les répercussions des événements parisiens entre Alpes et Pyrénées. Actes du colloque des 18 et 19 septembre 1998, Université Paul Valéry-Montpellier 3, Montpellier, Centre d'histoire moderne et contemporaine de l'Europe méditerranéenne et de ses périphéries, 1999, pp. 261-274.

Peter MAC PHEE, The Politics of the Rural Life, Political Mobilization in the French Countryside 1846-1852, Oxford, Clarendon Press, 1992, 310 p.

Ted. W. MARGADANT, French Peasants in Revolt. The Insurrection of 1851, Princeton N. J., Princeton University Press, 1979, XXIV-379 p. [contient une importante bibliographie sur les départements]

Bernard MÉNAGER, Les Napoléon du Peuple, Paris, Éditions Aubier, 1988, 445 p.

John M. MERRIMAN, The Agony of the Republic, The Repression of the Left in Revolutionary France, 1848-1851, New Haven Conn., Yale University Press, 1978, 298 p.

J. G. PRAT, Les exploits du 2 décembre, récits d'histoire du XIXe siècle. Deuxième série. Le dernier combattant. Un suspect sous le Second Empire, Paris, Dentu, 1889, in 18, VII-245 p.

Édith ROZIER-ROBIN, "Le souvenir du 2 décembre dans la mémoire républicaine 1868-1901", dans 1848, Révolutions et mutations au XIXe siècle, n 1, 1985, pp. 87-113.

Fernand RUDE, "Mourir à Douera", dans Le Mouvement social, $\mathrm{n}^{\circ} 161$, octobre-décembre 1992, pp. 7-24.

Axel SANSON, La mémoire du passé, l'image de la barricade au XIXe siècle, Mémoire de maîtrise sous la direction de Ségolène Le Men, Université Paris 10, 1999.

Charles SEIGNOBOS, "Les opérations des commissions mixtes en 1852", dans La Révolution de 1848, tome 6, mai-juin 1909, pp. 59-67.

Iouda TCHERNOFF, Le parti républicain au coup d'État et sous le Second Empire, Paris, Éditions Pedone, 1906, $671 \mathrm{p}$.

Guy THUILLIER et Vincent WRIGHT, "Pour l'histoire du coup d'État, une source à exploiter : les dossiers des pensionnés du 2 décembre 1851", dans Le Mouvement social, $\mathrm{n}$ - 94, janvier-mars 1976, pp. 97-106.

Georges WEILL, Histoire du parti républicain en France, 1815-1870, Paris, Librairie Félix Alcan, 1900, 552 p. ; réimpression Genève, Slatkine reprint, 1980, 431 p.

Vincent WRIGHT, "The Coup d'Etat of December 1851. Repression and the Limits of the Repression", dans Roger PRICE [ed.], Revolution and Reaction: 1848 and the Second French Republic, Londres, Croom Helm, 1975, pp. 303-333. 


\section{BIBLIOGRAPHIE DÉPARTEMENTALE, POSTÉRIEURE À 1880, CLASSÉE PAR GRANDES RÉGIONS ET DÉPARTEMENTS \\ Paris et bassin parisien}

Seine

Patrick CHAMOUARD, Paris en décembre 1851, le comportement des représentants montagnards, Mémoire de maitrise sous la direction de Philippe Vigier, Nanterre, 1974, 213 p.

Gilles FRANCKAUSER, Les insurgés de Paris et ses faubourgs devant une justice militaire, civile et politique après le coup d'État de Louis-Napoléon Bonaparte du 2 décembre 1851, Mémoire de maîtrise sous la direction de Nicole Piétri, Université de Poitiers, 1994.

Yonne

H. MARCOUX, "L'insurrection républicaine toucyoise du 6 décembre 1851", dans Actes du 39 congrès de l'Association bourguignonne des sociétés savantes, Toucy, 1968, pp. 25-30.

Jean SÉGUIN, "À propos de l'insurrection à Toucy et en Puisaye de décembre 1851", dans Bulletin de l'association d'études, de recherches et de protection du vieux Toucy, n 2-3, 1962, pp. 10-21 et 32-35.

C. LÉVY, "À propos du coup d'État de 1851 dans l'Yonne. Aux sources de l'opinion républicaine sous la deuxième République", dans Annales de Bourgogne, $\mathrm{n}^{\circ} 25,1953$, pp. 185-193.

Denis MARTIN, Les rouges de l'Yonne en 1851, Esquisse d'un profil, Mémoire de maîtrise, Université Paris 10, 1995.

Nord

Max BRUCHET, "Le coup d'État de 1851 dans le département du Nord", dans Revue du Nord, tome 9, 1925, pp. 81-114.

Bourgogne

A. JACOTIN, "Notes sur le coup d'État du 2 décembre 1851 en Côte d'Or", dans Annales de Bourgogne, tome 13, 1941, pp. 73-96.

Pierre LÉVÊQUE, Une société en crise, la Bourgogne au milieu du XIXe siècle 1846-1852, Paris, Éditions de l'École des hautes études en sciences sociales/Librairie J. Touzot, 1983, V-592 p.

Centre

Allier

Jean CORNILLON, Le Bourbonnais en décembre 1851. Le Coup d'État, Fumoux, Cusset imprimeur, 1903, XI-383 $\mathrm{p}$.

Annick GIROZ, La résistance de l'Allier au coup d'État de Louis-Napoléon Bonaparte, Mémoire de maîtrise sous la direction de Philippe Vigier, Université Paris 10, 1984, 92 fo.

Paul HENNEQUIN, Relation des événements survenus dans l'Allier en 1851, Insurrections du Cher et de la Nièvre avant le 2 décembre, Moulins, Fondard, s.d., in $8^{\circ}, 64 \mathrm{p}$.

Georges ROUGERON, Y.-H. MONCEAU, A. LEGUAI et M. DARGAUD, De la révolution de février au 2 décembre 1851. La révolution de 1848 à Moulins et dans le département de l'Allier, Moulins, Comité départemental du centenaire de la révolution de 1848/A. Pottier, 1950, 300 p.

Georges ROUGERON, "La résistance au coup d'État dans le département de l'Allier", dans La Révolution de 1848, 1935, pp. 341-352.

Georges ROUGERON, "La terreur bonapartiste dans le département de l'Allier après le coup d'État", dans La Révolution de 1848, 1937-38, pp. 155-171.

Cher

René GANDILHON, "Documents sur l'émeute survenue à Saint-Amans, Cher, le 3 décembre 1851", dans La Révolution de 1848, 1936-37, pp. 44-48. 
Ferdinand PÉLOILLE, "Le procès des Mariannes du département du Cher (1851-52)", dans Union des sociétés savantes de Bourges, mémoires, n 8, 1959-60, pp. 122-158.

Creuse

Jean BOUDARD, "La répression policière en Creuse au lendemain du coup d'État de 1851", dans Mémoires de la Société de la Creuse, 1953, pp. 429-35.

Nièvre

M. AUTENZIO, La résistance au coup d'État du 2 décembre dans la Nièvre, Mémoire de maîtrise sous la direction de Philippe Vigier, Université d'Orléans-Tours, 1970.

Clamecy et le coup d'État de 1851, Clamecy, Société scientifique et artistique de Clamecy, 1951.

Coup d'État du 2 décembre 1851, les insurgés de Clamecy et de la Nièvre, Actes du colloque tenu à Clamecy le 24 mai 1997, Clamecy, Société scientifique et littéraire de Clamecy, 1999, 315 p.

Jean-Claude MARTINET, Clamecy et ses flotteurs, de la monarchie de Juillet à l'insurrection des "Marianne", 1830-1851, Précy-sous-Thil, Éditions de l'Armançon, 1995 (1ère édition 1975), $151 \mathrm{p}$.

"Souvenir de Marc-Etiennne Piron, détenu politique de décembre 1851", dans La Révolution de 1848, tome 4, 1907-1908, pp. 106-111.

Guy THUILLIER, "Les souffrances des prisonniers de Clamecy (décembre 1851-mars 1852)", dans Le Mouvement social, n 161, octobre-décembre 1992, pp. 25-42.

Simone WAQUET, "Tristes destins, Le sort des victimes de 1851 dans l'arrondissement de Clamecy", dans Société d'histoire de la Révolution de 1848, Répression et prison politiques en France et en Europe au XIXe siècle, Paris, Éditions Créaphis, 1990, pp. 97-112.

Simone WAQUET, "Une petite ville intrépide, Clamecy, au printemps de 1851", dans Bulletin de la Société scientifique et artistique de Clamecy, n² 12, 1988, pp. 51-67.

Simone WAQUET, "Un voyage sans retour, la traversée des déportés de 1851 vers Cayenne d'après Numa Millelot", dans Bulletin de la Société scientifique et artistique de Clamecy, $\mathrm{n}^{\circ} 14$, 1991, pp. 56-76.

Puy-de-Dôme

Jacques VALENTIN, "Les répercussions du coup d'État du 2 décembre 1851 dans le département du Puy-de-Dôme", dans Revue d'Auvergne, n 65, 1951, pp. 145-162.

Haute-Vienne

Jean MARION et Christian PALVADEAU, "L'insurrection de Linards, 6 décembre 1851", Société historique du canton de Chateauneuf-La Forêt, janvier 1998, 243 p.

Est

Ain

Hippolyte DRUARD, Une page de l'histoire du 2 décembre. Le Coup d'État dans l'Ain, Bourg, Le Réveil de l'Ain, 1885 , in $8^{\circ}, 36 \mathrm{p}$.

P.CARON, "L'État politique du département de l'Ain en décembre 1852", dans La Révolution de 1848, 1904-1905, tome 1, pp. 93-99 et 140, tome 2, p. 318.

Doubs

Roger MARLIN, L'épuration politique dans le Doubs à la suite du coup d'État du 2 décembre 1851, Dôle, 1958, imprimerie Chazelle, 40 p.

Jura

Pierre MERLIN, "Le coup d'État du 2 décembre 1851 dans le Jura", dans Société d'émulation du Jura, Travaux, 1994, Lons le Saunier, 1996, pp. 155-182.

Pierre MERLIN, "Le Coup d'État du 2 décembre 1851 dans le Jura, la répression, les hommes, l'exil", dans Société d'émulation du Jura, Travaux, 1995, Lons le Saunier, 1997, pp. 281-313.

Revue d'histoire du XIXe siècle, 22 | 2001 
Pierre MERLIN, "Poligny et l'idée démocratique sous la Seconde République, février 1848-2 décembre 1851", dans Patrimoine Polinois, $\mathrm{n}^{\circ}$ 15, 2000. pp. 91-101.

Haut-Rhin

P. MULLER, "Autour du coup d'État dans le Haut-Rhin", dans La Révolution de 1848, 1909-10, pp. 197-212.

Région alpine

Philippe VIGIER, La Seconde République dans la région alpine. Tome 2: Les Paysans, Paris, Presses universitaires de France, 1963, 527 p.

Association 1851-2001, Provence 1851. Une insurrection pour la République, Gap, 2000, 238 p.

Basses-Alpes

Giselle ROCHE-GALLOPINI, Saint-Étienne les Orgues et la gloire de la Montagne, Mane, Éditions Alpes de Lumières, 1994, 172 p.

G. MANTOY, "Le soulèvement de décembre 1851 dans la vallée de Barcelonnette", dans Annales de Haute-Provence, Digne, n 304, 1987.

Paul VARCIN, "Contribution à l'histoire du Coup d'État du 2 décembre 1851 dans les Basses Alpes. Jean-François Ailhaud et quelques autres instituteurs", dans Annales de Haute-Provence, Digne, $\mathrm{n}^{\circ}$ 301, 1986.

Hautes-Alpes

Henri BLET, "La résistance au coup d'État du 2 décembre 1851 dans le département des Hautes-Alpes", dans Procès-verbaux de la société dauphinoise d'ethnologie, 1951, pp. 26-37.

Sud-Ouest

Aveyron

André ANCOURT, "Le coup d'État du 2 décembre et ses répercussions à Villefranche", dans Revue du Rouergue, 1952-53, pp. 456-73 et 177-196.

André ANCOURT, Des journées de février 1848 au coup d'État du 2 décembre 1851. Esquisse d'une histoire politique et sociale de Villefranche-du-Rouergue sous la Seconde République, Rodez, Carrère imprimeur, 1957, $110 \mathrm{p}$.

Deux Sèvres

Marcel NÉSI, "La résistance au coup d'État du 2 décembre dans les Deux-Sèvres", dans La Révolution française, juillet, 1914, pp. 63-68.

Haute-Garonne

Alphonse BRÉMOND, Histoire du coup d'État dans le département de la Haute-Garonne (1851-52), Toulouse, Hébrail Durand et cie 1870, in 18, 180 p.

Gers

Jean DAGNAN, Le Gers sous la Seconde République. Tome 2 : Le Coup d'État et la répression dans le Gers (décembre 1851-décembre 1852), Auch, Imprimerie F. Cocharaux, 1929, in 8 IX-590 p.

Georges DUTOIS, Le Crime du 2 décembre dans le Gers, Auch, de Capin, 1902, in 18, 49 p.

Lot-et-Garonne

Léo DELBERGE, "Le coup d'État du 2 décembre 1851 en Lot-et-Garonne, d'après Eugène Ténot, rédacteur du Siècle", dans Revue de l'Agenais, n 83, 1957, pp. 91-101.

Jean-Alfred NEUVILLE, 2 décembre 1851, proscription de Marmande (Lot-et-Garonne), Agen, de Bonnet et fils, 1882, in 16, $479 \mathrm{p}$.

Tarn

André ARMENGAUD, "Coup d'État et plébiscite dans le département du Tarn", dans Annales du Midi, janvier 1952, pp. 41-47.

Vallée du Rhône

Ardèche

Guy-Jean ARCHÉ, L'espoir au cœur. L'insurrection en Drôme-Ardèche en 1851, Poët-Laval, 
Curandera, 1981.

Élie REYNIER, La Seconde République dans l'Ardèche 1848-1852, Privas, Maison de l'Enfance/ Imprimerie de L.Volle, 1948; réédition: Privas, Fédération des œuvres laïques de l'Ardèche, 1998, 288 p.

Drôme

M. FAURE, Le coup d'État dans la Drôme. Discours du 11 décembre 1910. Inauguration à Crest du monument commémoratif de la résistance de la Drôme au coup d'État du 2 décembre 1851, Valence, Imprimerie de C. Legrand, 1910, $32 \mathrm{p}$.

Pierre GAUDIN et Claire REVERCHON, "Les insurgés de la Drôme, images héritées, images transmises", dans Société d'histoire de la révolution de 1848 et des révolutions du XIXe siècle, Philippe VIGIER [dir.], Répression et prisons politiques en France et en Europe au XIXe siècle, Paris, Éditions Créaphis, 1990, pp. 159-170.

Pierre GAUDIN et Claire REVERCHON, "Une prison de longue durée, la tour de Crest, symbole de la répression antihuguenote et antirépublicaine dans le légendaire historique drômois", dans Société d'histoire de la révolution de 1848 et des révolutions du XIXe siècle, Philippe VIGIER [dir.], Maintien de l'ordre et polices en France et en Europe au XIXe siècle. Actes du colloque de Paris et Nanterre, 8-10 décembre 1983, Paris, Éditions Créaphis, 1987, pp. 213-232.

L. GOUDIN, Livre d'or des victimes du coup d'État de 1851 et de la loi de sûreté générale de 1858, Valence, 1883.

Ted. W. MARGADANT, "Modernisation and Insurgency in December 1851, A Case Study of the Drôme", dans Roger PRICE [ed.], Revolution and Reaction: 1848 and the Second French Republic, Londres, Croom Helm, 1975, pp. 254-279.

Roger PIERRE, Ah! Quand viendra la Belle. Résistants et insurgés de la Drôme, 1848-1851, Valence, Éditions Notre temps, 1981, 248 p.

Loire

Claude LATTA, "La répression antirépublicaine dans la région de Montbrison après le coup d'État du 2 décembre 1851", dans Bulletin de la Diana, société, historique et archéologique du Forez, 1990.

Cyril PAUTRAT, La résistance au coup d'État en décembre 1851 à Saint-Étienne, Mémoire de maîtrise sous la direction de Didier Nourrisson, Université Jean Monnet-Saint-Étienne, 1999.

Rhône

Melle VERGEZ-TRICOM, "Les événements de décembre 1851 à Lyon", dans La Révolution de 1848, tome 16, 1920-21, pp. 226-253.

Vaucluse

Aimé AUTRAND, "Commémoration de la résistance vauclusiennne au coup d'État de décembre 1851", dans Annuaire administratif et statistique du Vaucluse, 1951, pp. 279-394.

Pierre SIMONI, "La lutte contre l'insurrection de décembre1851; un titre à la Légion d'honneur, l'exemple vauclusien", dans Provence historique, fascicule 202, octobredécembre 2000, pp. 475-493.

Sud-Est

Bouches-du-Rhône

Michèle BOCQUET, "Les proscrits de l'insurrection du 2 décembre 1851 d'après les dossiers de pension de la série F 15 des Archives nationales dans le département des Bouches-du-Rhône", Mémoire de maitrise sous la direction de Maurice Agulhon, Université Paris 1, 1977.

Hubert GAY, "La résistance au coup d'État du 2 décembre 1851 dans la région de l'étang 
de Berre", dans Roland ANDRÉANI et Marianne LEULLIEZ [dir.], De la Révolution au coup d'État (1848-1851). Les répercussions des événements parisiens entre Alpes et Pyrénées. Actes du colloque des 18 et 19 septembre 1998, Université Paul Valéry-Montpellier 3, Montpellier, Centre d'histoire moderne et contemporaine de l'Europe méditerranéenne et de ses périphéries, 1999, p. 201-212.

Var

Maurice AGULHON, La République au village, Paris, Éditions Plon, 1970, 543 p.

Maurice AGULHON et Yvonne KNIBIELHER, "Lord Brougham, juge de l'insurrection varoise de décembre 1851", dans Provence historique, tome 16, fascicule 64, 1966, pp. 177-186.

Charles DUPONT, Les républicains et les monarchistes dans le Var en décembre 1851, Paris, Germer-Baillière, 1883, in 18, 275 p.

Alexandre GARIEL, Le coup d'État de décembre 1851 dans le Var, Draguignan, Gimbert fils, 1878.

Victor FOURNIER, Le coup d'État de 1851 dans le Var, Draguignan, Olivier Joulian, 1928.

Jean-Jacques LETRAIT, "L'insurrection dans le Var en 1851", dans Bulletin de la Société d'Études de Draguignan, nouvelle série, tome 12, 1967.

Frédéric NÉGREL, Clandestinité et réseau républicain dans le Haut-Var. La société secrète montagnarde d'Artignosc (1849-1851), Mémoire de maîtrise sous la direction de Jean-Marie Guillon, Université d'Aix-en-Provence, 2000 ; édition : Aix, Gemenos, 2001, 320 p.

Dominique SAMPIERI, La faux et le fusil. Instauration, défense, et renversement de la Seconde République à Hyères, 1848-1851, Hyères, chez l'auteur, 1999, 370 p.

Comté de Nice

André COMPAN, "Les réfugiés politiques provencaux dans le comté de Nice après le coup d'État du 2 décembre 1851", dans Provence historique, tome 7, fascicule 27, janvier-mars 1957.

Un proscrit varois de décembre 1851 dans le comté de Nice, le docteur César Provençal (1848-1868), dans Nice historique, $\mathrm{n}^{\circ}$ 3, octobre-décembre 1953.

\section{Bas-Languedoc et Roussillon}

Aude

Jean-François JEANJEAN, "Le coup d'État du 2 décembre 1851 dans le département de l'Aude", dans La Révolution de 1848, 1924-25, pp. 161-180.

Christopher E. GUTHRIE, "Reaction to the Coup d'État in the Narbonnais. A Case Study of Popular Political Mobilization and Repression during the Second Republic", dans French Historical Studies, volume 13, n 1, 1983, pp. 18-46.

Claude MARQUIÉ et Jean RAFFANEL, "La répression dans l'Aude après le coup d'État du 2 décembre 1851", dans Roland ANDRÉANI et Marianne LEULLIEZ [dir.], De la Révolution au coup d'État (1848-1851). Les répercussions des événements parisiens entre Alpes et Pyrénées. Actes du colloque des 18 et 19 septembre 1998, Université Paul Valéry-Montpellier 3, Montpellier, Centre d'histoire moderne et contemporaine de l'Europe méditerranéenne et de ses périphéries, 1999, pp. 213-226.

Gard

Jean-Pierre GOIRAND, Documents sur le coup d'État de 1851 dans le Gard, Alès, 1881, 191 p.

J. FONTANE, "Autour du coup d'État, souvenirs d'un paysan, recueillis par A. Detrez", dans La Révolution de 1848, juillet 1909, pp. 165-170.

Raymond HUARD, Le mouvement républicain en Bas-Languedoc, 1848-1881, Paris, Presses de la Fondation nationale des sciences politiques, 1982, $520 \mathrm{p}$.

Raymond HUARD, "Aux origines d'un thème républicain : la défense de la République. la 
riposte au coup d'État de 1851 dans le Gard", dans La République en Languedoc-Roussillon 1792-1958, Nîmes, Société d'histoire moderne et contemporaine de Nîmes, 1993, pp. 213-227.

Hérault

Maurice AGULHON, Jean SAGNES, Claire TORREILLES et Raymond HUARD, "Quarantehuitards et déportés. Les républicains de l'Hérault au milieu du XIXe siècle", dans Études sur Pézenas et l'Hérault, tome 12,1981-2, 54 p.

Émile APPOLIS, "La résistance au coup d'État du 2 décembre 1851 dans l'Hérault", dans Actes du 77e congrès des sociétés savantes, Histoire moderne et contemporaine, Grenoble, 1952, pp. 487-304.

F. CHARRAS, Témoignages sur le coup d'État du 2 décembre dans la vallée de Saint-Pons de Thomières, Bulletin de la Société archéologique et historique des Hauts cantons de l'Hérault, $n$ -19, 1996, p. 89-107.

Jean COUBÈS, "Le Coup d'État du 2 décembre 1851 et ses répercussions dans le département de l'Hérault", Archives départementales de l'Hérault, 1980, 33 documents.

Robert FERRAS, "Un déporté du 2 décembre, Jean Pech de Capestang", dans Mémoires de la société archéologique de Montpellier, tome 14, 1971.

Robert FERRAS, "Les suites du Coup d'État du 2 décembre 1851 dans un village du Biterrois, Capestang", dans Actes du 43e congrès de la fédération historique du Languedoc méditerranéen et du Roussillon, 1970, Montpellier, 1971, pp. 369-374.

Marcel HÉNAUX, Les victimes de décembre 1851 dans l'Hérault: devenir des inculpés et des condamnés, Mémoire de maitrise sous la direction de Raymond Huard, Université Montpellier 3, 1987.

G. LAURIBEL, "Pézenas et le coup d'État de Louis-Napoléon Bonaparte", dans Connaissance de Pézenas, Pézenas, G. Domens 1960, 84 p.

J. MONTELS, La justice de l'ordre en 1851. La vie et la mort d'André Abel Cadelard, Béziers, 1881.

A. E. MOULIN, Un républicain martyr, Casimir Péret, (Le Coup d'État à Béziers, la déportation, la mort), Montpellier, Causse Graille, Castelnau, 1937.

Jean-Claude RICHARD, "Bédarieux, le coup d'État du 2 décembre 1851 et les partis de l'ordre; une médaille locale de 1851-52", dans Bulletin de la Société archéologique et historique des Hauts cantons de l'Hérault, $n^{\circ}$ 17, 1994, p. 121-154.

Jean-Claude RICHARD, "Les monuments érigés dans l'Hérault en l'honneur des victimes du coup d'État", dans Roland ANDRÉANI et Marianne LEULLIEZ [dir.], De la Révolution au coup d'État (1848-1851). Les répercussions des événements parisiens entre Alpes et Pyrénées. Actes du colloque des 18 et 19 septembre 1998, Université Paul Valéry-Montpellier 3, Montpellier, Centre d'histoire moderne et contemporaine de l'Europe méditerranéenne et de ses périphéries, 1999,, p. 237-260.

Jean SAGNES, "Le coup d'État dans l'Hérault, par Blayac, tailleur à Mèze", dans Bulletin de la société d'études scientifiques de Sète et sa région, $\mathrm{n}^{\circ}$ 12-13, 1983, pp. 169-181.

Jean SAGNES, "Les opposants au coup d'État devant le conseil de guerre, l'affaire de Béziers", dans Roland ANDRÉANI et Marianne LEULLIEZ [dir.], De la Révolution au coup d'État (1848-1851). Les répercussions des événements parisiens entre Alpes et Pyrénées. Actes du colloque des 18 et 19 septembre 1998, Université Paul Valéry-Montpellier 3, Montpellier, Centre d'histoire moderne et contemporaine de l'Europe méditerranéenne et de ses périphéries, 1999, pp. 227-236.

Louis THOMAS, "Montpellier en 1851, le coup d'état du 2 décembre 1851", dans Monspeliensa, tome 1, $\mathrm{n}^{\circ}$ 3, 1933, pp. 11-12.

M. VILLEMAGNE, Événements politiques à Saint-Thibéry, (Hérault), en 1851-1852, Saint-Pons, 
M. Maraval, 1903, in $8^{\circ}$, IV-47 p.

Pyrénées-orientales

André BALENT, "La résistance au coup d'État du 2 décembre 1851 en Roussillon et la répression gouvernementale", dans Massana, n² 20, 1973, pp. 396-408.

Horace CHAUVET, Histoire du parti républicain dans les Pyrénées orientales de 1830 à 1877, d'après des documents et des souvenirs inédits, Perpignan, Imprimerie de L'Indépendant, 1909, $296 \mathrm{p}$.

Peter MAC PHEE, Les semailles de la République dans les Pyrénées orientales 1846-1852, Perpignan, Éditions de l'Olivier, 1995, 507 p.

Ouest

Finistère

Georges Michel THOMAS, "Le coup d'État du 2 décembre 1851 dans le Finistère et à Brest [...]", dans Actes du 107e congrès des Sociétés savantes. Brest, 1982. Histoire moderne et contemporaine, tome 2, Paris, Éditions du Comité des travaux historiques et scientifiques, 1984, pp. 103-115.

Maine et Loire

Abbé L. COURANT, "L'Anjou et le coup d'État du 2 décembre 1851", dans L'Anjou historique, 1953, pp. 29-44.

Manche

Léon DERIÈS, "Autour du coup d'État du deux décembre dans la Manche", dans Notices et documents publiés par la société d'agriculture et d'archéologie de la Manche, $\mathrm{n}^{\circ}$ 41, 1929, pp. 263-267.

Morbihan

CORGNE, "Le coup d'État du 2 décembre 1851 et le Morbihan", dans Bulletin de la société polymathique du Morbihan, 1961, pp. 44-45 et 51-52.

Sarthe

Corinne CHABLE, La Suze-sur-Sarthe et la réaction au coup d'État de Napoléon III en 1851, Mémoire de maîtrise sous la direction de Philippe Vigier, Université Paris 10, 1990.

Léon GUYON, Épisodes du coup d'État en 1851 dans la Sarthe. Une évasion. Troisième et dernière partie de l'étude historique sur Charles Granger et son époque, préface d'A. Hamonet, Le Mans, Librairie de Saint-Denis, 1905, in 16, 176 p.

Léon Guyon, Les Martyrs du droit et de la liberté dans la Sarthe, récit des événements de décembre 1851 dans notre département, Paris, Charavay, 1883, in 18, 443 p.

Algérie

Marcel EMERIT [dir.], La révolution de 1848 en Algérie: mélanges d'histoire, Paris, Éditions Larose, 1949, 191 p.

\section{OUVRAGES SUR LES COUPS D'ÉTAT EN GÉNÉRAL}

Maurice AGULHON, Coup d'État et République, Paris, Presses de Sciences-Po, 1997, 97 p. Jean DUMONT [dir.], Les coups d'État, Paris, Club des amis du livre, 1963.

Pierre LEROY, "Coup d'État" dans Olivier Duhamel et Yves Mény [dir.], Dictionnaire constitutionnel, Paris, Presses universitaires de France, 1992, 1112 p.

Edward N. LUTTWAK, Le coup d'État, théorie et pratique, Paris, Éditions Robert Laffont, 1969, in $8^{\circ}, 267 \mathrm{p}$.

Edward N. LUTTWAK, Coup d'État, mode d'emploi, Paris, Éditions Odile Jacob, 1996285 p.

Louis MARIN, "Pour une théorie baroque de l'action politique", introduction, dans Gabriel NAUDÉ, Considérations politiques sur les coups d'État, Paris, Éditions de Paris, 1989, 221 p.

Onésime MONPROFIT, Les Coups d'État, histoire et théorie, 18 brumaire, 1830, 2 décembre, Paris, 
G. Carré,1887, 161 p.

Jeff TALMAN, Aux origines du bonapartisme : le coup d'État 1836 et 1840, Mémoire de maîtrise sous la direction de Francis Démier, université Paris 10, 1995149 p.

VII. GUVRES LITTÉRAIRES AYANT POUR SUJET, AU MOINS PARTIELLEMENT, LE COUP D'ÉTAT DE 1851

Louis ANDRÉ et Jean BOSC, La haine d'un gardian, roman de mours languedociennes, Montpellier, librairie du Petit méridional, 1885, grand in $8^{\circ}$, IV-307 p.

Gaston BELTRAME, Le Còp d'estat de 1851, Toulon, Éditions Rescontre/Centre dramatique occitan de Provence, 1974, 84 p. [pièce de théâtre]

Jacques BONNET, Le cheval cabré ou la vie de Jules Sibilat (1848-1897), militant ouvrier, compagnon et anarchiste, Montpellier, Éditions Max Chaleil, 1992.

Jules BOULABERT, Le Guet apens, Paris, Collomb et Brûlé, grand in folio, 1880.

Jean BRUNO [Jean VAUCHERET], Brisefer l'insurgé, Histoire populaire du deux décembre, Paris, J. Brouillet, 1873.

Jules CLARETIE, Le petit Jacques, Paris, Laffite, 1910, 112 p. [réédition d'un ouvrage publié sous le titre de Noël Rambert en 1872]

Daniel HÉNARD, Le grand flot, nouvelles, Paris, Éditions Baudinière, 1979.

André NEYTON, La farandole de la liberté, Marseille, Éditions Autres temps, 2001, 85 p.

Jean RAMBAUD, La Provence insurgée, Frédéric Arnaud, 1851, Marseille, Éditions Autres temps, 1995. [réédition d'un ouvrage publié en 1974 sous le titre de Frédéric Arnaud]

Yves ROUQUETTE, Lengadoc Roge, Los enfants de la Bona, Toulouse, Institut d'Estudis Occitans, 1984.

Jean SICCARDI, Fernand Martin, dit Martin Bidouré, Paris, Presses de la cité, 2000.

Luc WILLETTE, [Luc BIHL], Et la montagne fleurira, Paris, Éditions Denoël, 1975.

Émile Zola, La fortune des Rougon, Paris, Librairie internationale A. Lacroix, Verboekhoven et cie, 1870-1871.

\section{FILMS}

Ils se levèrent pour la République, Christian Philibert, Copsi, 2000.

\section{ANNEXE SUR LES OUVRAGES COMPLÉMENTAIRES CITÉS DANS L'INTRODUCTION}

Histoires de la Seconde République accordant une place significative au coup d'État Victor PIERRE (1873-1878), Pierre de LA GORCE (1887), G. RENARD (1905), Charles SEIGNOBOS (1920), Jean DAUTRY (1957), Émile TERSEN (1957), Philippe VIGIER (1967), Louis GIRARD (1968), Maurice AGULHON $(1973,1992)$, Inès MURAT (1987), Sylvie APRILE (2000).

Biographies de Louis Napoléon ou Napoléon III ou des protagonistes du coup d'État Elles sont très nombreuses et certaines figurent dans la bibliographie ci dessus. On retiendra particulièrement, soit pour la qualité documentaire, soit pour l'interprétation du rôle de Louis-Napoléon, les ouvrages de Paul GUÉRIOT (1934), Heinrich MULLER (en allemand, 1961), Georges ROUX (1969), W. H. C. SMITH (en anglais, 1972), Louis GIRARD (1984). Parmi les protagonistes du coup d'État, c'est Morny surtout qui a attiré l'attention des biographes: Marcel BOULENGER (1925), Claude DUFRESNE (1983), Jean-Marie ROUART (1995).

\section{Travaux départementaux ou régionaux}

Enfin, parmi les travaux non cités dans la bibliographie ci-dessus, on mentionnera particulièrement ceux de André ARMENGAUD (Aquitaine), Alain CORBIN (Limousin), 
Michel DENIS (la Mayenne), François IGERSHEIM (Bas-Rhin), Christiane MARCILHACY (Loiret), Auguste RIVET (Haute-Loire), Jean-François SOULET (Pyrénées centrales), JeanLuc MAYAUD (Doubs), Marcel VIGREUX (Morvan).

\section{ABSTRACTS}

La bibliographie que nos présentons ci dessous s'efforce de cerner au plus près ce qui a été écrit sur le Coup d'État du 2 décembre 1851 depuis l'événement jusqu'à nos jours.

INDEX

Mots-clés: Bibliographie, Napoléon III 